\title{
Compatibilidad dimensional entre los conos accesorios de gutapercha y los espaciadores
}

Dimensional compatibility between accessory gutta-percha cones and spreaders

\section{Abreu-Rodríguez, Rixio* Naval-Esteve, Victoria* Montesinos-Vidal, Beatriz * Pallarés-Sabater, Antonio **}

* Licenciado(a) en Odontología, Master en Patología y Terapéutica Dental. ** Profesor Asociado del Departamento de Estomatología de la Universidad de Valencia - España.

Universidad de Valencia Facultad de Medicina y Odontología, Departamento de Estomatología: Unidad Docente de Patología y Terapéutica Dental - Master de Patología y Terapéutica Dental.

\section{Correspondencia}

Antonio Pallarés Sabater.

Gasco Oliag No 1. Universidad de Valencia.

Clínica Odontológica - Unidad de PTD.

Master de Patología y Terapéutica

Dental.

46010 -Valencia - España

\begin{abstract}
Resumen: Objetivo: Evaluar la compatibilidad dimensional entre los conos de gutapercha y los espaciadores. Material y método: Se seleccionaron aleatoriamente conos de gutapercha y espaciadores de diferentes tamaños y fabricantes (Dentsply - DeTrey, Dentsply Maillefer y Boston Dental Scientific). Se conformaron 26 grupos (n: 10). Cada una de las muestras se observó con un microscopio óptico (50 - 100X), y se midió D1 y D2. Los resultados se sometieron a un análisis estadístico descriptivo. Resultados: De acuerdo con nuestros datos, existe variabilidad dimensional entre los conos de gutapercha de un mismo tamaño, los espaciadores y las puntas de gutapercha y los espaciadores entre sí, aunque las diferencias entre este último grupo son menores. Conclusión: Existen discrepancias dimensionales entre los conos de gutapercha y los espaciadores, las cuales podrían causar inconvenientes durante la obturación tridimensional del sistema de conductos radiculares y propiciar un sellado apical incorrecto.
\end{abstract}

Palabras clave: Obturación de conductos, Espaciadores, Gutapercha, Instrumentos endodóncicos.

Abstract: Objetive: This study evaluated the dimensional compatibility between guttapercha cones and spreaders. Material and method: Gutta-percha cones and spreaders of different sizes and brands were randomly selected (Dentsply / DeTrey, Dentsply / Maillefer and Dental Boston Scientific). 26 groups were made ( $\mathrm{n}$ : 10). Each sample was observed with an optical microscope (50-100X), and measured at $D_{1}$ and $D_{2}$. The results were sujected to a descriptive statistical analysis. Results: According to our data, there was dimensional variability among gutta-percha cones of a same size, spreaders and guttapercha points and among spreders, although the differences in the last group were smaIler. Conclusion: There are dimensional discrepancies between gutta-percha cones and spreaders, which could cause disadvantages during the three-dimensional root canal filling and leaf to an incorrect apical sealing.

Key words: Root canal filling, Spreaders, Gutta-percha, Root canal instruments.

\begin{tabular}{ccc}
\hline Fecha recepción & Fecha última revisión & Fecha aceptación \\
$29-12-2003$ & $28-07-2004$ & $4-11-2004$ \\
\hline
\end{tabular}

BIBLID [1138-123X (2004)9:6; noviembre-diciembre 613-736]

Abreu-Rodríguez R, Naval-Esteve V, Montesinos-Vidal B, Pallarés-Sabater A. Compatibilidad dimensional entre los conos accesorios de gutapercha y los especiadores. RCOE 2004;9(6):645-652. 


\section{Introducción}

El estadío final del tratamiento endodóncico consiste en la obturación tridimensional del sistema de conductos radiculares y de sus complejas irregularidades anatómicas con agentes selladores no irritantes (cemento y gutapercha), además de la restauración definitiva'.

Con el paso de los años, han sido propuestas varias técnicas de obturación, aunque la más utilizada y aceptada a nivel mundial es la de condensación lateral, en la cual se utiliza un cono principal de un tamaño aproximado al diámetro de la lima apical maestra y varias puntas accesorias de gutapercha en conjunto con un cemento sellador ${ }^{2 *}$. En la técnica de condensación lateral el uso de los espaciadores es indispensable y debe crear un espacio suficiente que permita introducir el cono accesorio de gutapercha y su posterior adaptación.

Existen diferentes tipos de gutaperchas y espaciadores en el mercado odontológico. Las preferencias personales, la disponibilidad de los materiales y la conicidad del conducto radicular son algunas de las consideraciones que se deben tener en cuenta a la hora de seleccionar los instrumentos y materiales de obturación $n^{3.4}$.

La literatura sugiere que la compatibilidad dimensional entre los conos accesorios de gutapercha y los espaciadores influye significativamente en la calidad de la obturación del sistema de conductos radiculares; por lo tanto, todos los instrumentos y materiales de obturación deben estar estandarizados (norma ISO: Dental root canal obturating cones), para asegu-

\begin{tabular}{|ll|}
\hline & Tabla I: GIUpOS de esfudio \\
\hline Grupo (n: 10). & Muestras \\
\hline Boston XXF D1 & Conos de gutaperchas XXF / Boston Scientific \\
\hline Boston XXF D2 & Conos de gutaperchas XXF / Boston Scientific \\
\hline Boston XF D1 & Conos de gutaperchas XF / Boston Scientific \\
\hline Boston XF D2 & Conos de gutaperchas XF / Boston Scientific \\
\hline Boston F D1 & Conos de gutapercha F / Boston Scientific \\
\hline Boston F D2 & Conos de gutapercha F / Boston Scientific \\
\hline Dentsply XXF D1 & Conos de gutapercha XXF / Dentsply DeTrey \\
\hline Dentsply XXF D2 & Conos de gutapercha XXF / Dentsply DeTrey \\
\hline Dentsply XF D1 & Conos de gutapercha XF / Dentsply DeTrey \\
\hline Dentsply XF D2 & Conos de gutapercha XF / Dentsply DeTrey \\
\hline Dentsply F D1 & Conos de gutapercha F / Dentsply DeTrey \\
\hline Dentsply F D2 & Conos de gutapercha F / Dentsply DeTrey \\
\hline Dentsply 20 D1 & Conos de gutapercha ISO - 020 \\
\hline Dentsply 20 D2 & Conos de gutapercha ISO - 020 \\
\hline Dentsply 25 D1 & Conos de gutapercha ISO - 025 \\
\hline Dentsply 25 D2 & Conos de gutapercha ISO - 025 \\
\hline Dentsply 30 D1 & Conos de gutapercha ISO - 030 \\
\hline Dentsply 30 D2 & Conos de gutapercha ISO - 030 \\
\hline Espaciador A - D1 & Espaciadores digitales A \\
\hline Espaciador A - D2 & Espaciadores digitales A \\
\hline Espaciador B - D1 & Espaciadores digitales B \\
\hline Espaciador B - D2 & Espaciadores digitales B \\
\hline Espaciador C - D1 & Espaciadores digitales C \\
\hline Espaciador C - D2 & Espaciadores digitales C \\
\hline Espaciador D 11T D1 & Espaciador digito - palmar D 11T \\
\hline Espaciador D 11T D2 & Espaciador digito - palmar D 11T \\
\hline
\end{tabular}

rar, desde el punto de vista teórico, un sellado hermético y tridimensional y así evitar la microfiltración apical y los sucesivos cambios biológicos de los tejidos perirradiculares ${ }^{5}$.

Ingle en $1955^{6}$, fue el primero en reseñar que el diámetro creado por el espaciador, era, en general, menor y no se correspondía con el tamaño de la punta de gutapercha accesoria. Años más tarde Sampeck y cols también reconocieron la necesidad de establecer una correlación lógica entre los espaciadores y las puntas accesorias de gutapercha ${ }^{4}$.
Kerekes $^{\text {*** }}$ realizó una investigación donde comparó los instrumentos de uso común en endodoncia y los conos de gutapercha con la norma ISO estándar. Él observó que existía una considerable variación entre los conos de gutapercha. Sin embargo, Jerome y cols ${ }^{8}$ realizaron un estudio de microfiltración apical donde utilizaron diferentes conos de gutapercha y espaciadores no viendo diferencias significativas entre los grupos de estudio.

Por otro lado, algunos trabajos ${ }^{7 *+10}$ han comparado la relación in vitro entre los espaciadores y las puntas acce- 
sorias de gutapercha, advirtiendo discrepancias dimensionales significativas que podrían facilitar un sellado apical incorrecto y propiciar el fracaso del tratamiento endodóncico; por estas razones y además teniendo en cuenta la introducción constante de nuevos materiales e instrumentos en el área de la endodoncia decidimos realizar esta investigación in vitro con el propósito de determinar la compatibilidad dimensional entre los conos de gutapercha de diferentes fabricantes y los espaciadores digitales y digito-palmares.

\section{Material y método}

En la realización de este estudio se han utilizado los conos de gutapercha extrafina - extrafina (XXF), extrafina fina (XF) y fina - fina (F) de treinta milímetros $(\mathrm{mm})$ de longitud, de las casas fabricantes Dentsply - DeTrey / China y Boston Scientific/Korea, conos de gutapercha ISO - 020, 025 y 030 (Dentsply DeTrey / China) de $25 \mathrm{~mm}$ de longitud, espaciadores digitales A, B y C de $25 \mathrm{~mm}$ de longitud (Dentsply - Maillefer/Europe), además de los espaciadores digito palmares D 11T (Hu - Friedy/USA).

Se conformaron veintiséis grupos de estudio. Cada grupo estaba constituido por diez muestras (n:10). Los primeros dieciocho estaban integrados por conos accesorios de gutapercha, los cuales se diferenciaban entre sí por el tipo de gutapercha y el fabricante. Los grupos restantes (8) estaban constituidos por espaciadores digitales y digito - palmares (tabla 1). Todas las muestras se seleccionaron aleatoriamente y se manipularon de manera sistemática, con la finalidad de evitar sesgos estadísticos.

\begin{tabular}{|c|c|c|c|c|c|}
\hline \multicolumn{6}{|c|}{ Tabla 2: Medidas obtenidas en los grupos de estudio Il } \\
\hline Grupo & Recuento & Media & $\begin{array}{l}\text { Desviación } \\
\text { estándar }\end{array}$ & Mínimo & Máximo \\
\hline Boston XXF & 10 & 0.185 & 0.017 & 0.15 & 0.21 \\
\hline Boston XF & 10 & 0.179 & 0.021 & 0.14 & 0.21 \\
\hline Boston F & 10 & 0.169 & 0.017 & 0.14 & 0.19 \\
\hline Dentsply XXF & 10 & 0.114 & 0.019 & 0.10 & 0.15 \\
\hline Dentsply XF & 10 & 0.143 & 0.011 & 0.12 & 0.16 \\
\hline Dentsply F & 10 & 0.115 & 0.014 & 0.10 & 0.14 \\
\hline Dentsply 20 & 10 & 0.198 & 0.020 & 0.17 & 0.23 \\
\hline Dentsply 25 & 10 & 0.252 & 0.019 & 0.22 & 0.29 \\
\hline Dentsply 30 & 10 & 0.293 & 0.015 & 0.27 & 0.32 \\
\hline Espaciador A & 10 & 0.206 & 0.005 & 0.20 & 0.21 \\
\hline Espaciador B & 10 & 0.221 & 0.003 & 0.22 & 0.23 \\
\hline Espaciador C & 10 & 0.221 & 0.003 & 0.22 & 0.23 \\
\hline Espaciador D 11T & 10 & 0.251 & 0.003 & 0.254 & 0.26 \\
\hline
\end{tabular}

\begin{tabular}{|c|c|c|c|c|c|}
\hline \multicolumn{6}{|c|}{ Tabla 3: Medidas obtenidas en los grupos de estudio D? } \\
\hline Grupo & Recuento & Media & $\begin{array}{l}\text { Desviación } \\
\text { estándar }\end{array}$ & Mínimo & Máximo \\
\hline Boston XXF & 10 & 0.528 & 0.014 & 0.50 & 0.54 \\
\hline Boston XF & 10 & 0.573 & 0.026 & 0.55 & 0.62 \\
\hline Boston F & 10 & 0.837 & 0.031 & 0.76 & 0.88 \\
\hline Dentsply XXF & 10 & 0.512 & 0.020 & 0.48 & 0.54 \\
\hline Dentsply XF & 10 & 0.558 & 0.024 & 0.50 & 0.59 \\
\hline Dentsply F & 10 & 0.717 & 0.018 & 0.69 & 0.75 \\
\hline Dentsply 20 & 10 & 0.490 & 0.017 & 0.46 & 0.52 \\
\hline Dentsply 25 & 10 & 0.542 & 0.022 & 0.52 & 0.60 \\
\hline Dentsply 30 & 10 & 0.603 & 0.021 & 0.57 & 0.64 \\
\hline Espaciador A & 10 & 0.600 & 0 & 0.60 & 0.60 \\
\hline Espaciador B & 10 & 0.750 & 0 & 0.75 & 0.75 \\
\hline Espaciador C & 10 & 1 & 0 & 1 & 1 \\
\hline Espaciador D 11T & 10 & 0.800 & 0 & 0.800 & 0.800 \\
\hline
\end{tabular}

Todos los especímenes se observaron a cincuenta y cien aumentos $(50$ - 100X), con un microscopio óptico con lentes milimetrados (Nikon SMZ - 2T/Japón). En cada uno de los conos de gutapercha y espaciadores digitales y digito - palmares se midió el diámetro a un milímetro de la punta $\left(D_{1}\right)$, y a dieciséis milímetros, referencia estándar de los instrumentos endodóncicos que se conoce como D2.

De las muestras estudiadas se obtuvieron doscientas sesenta medidas en 


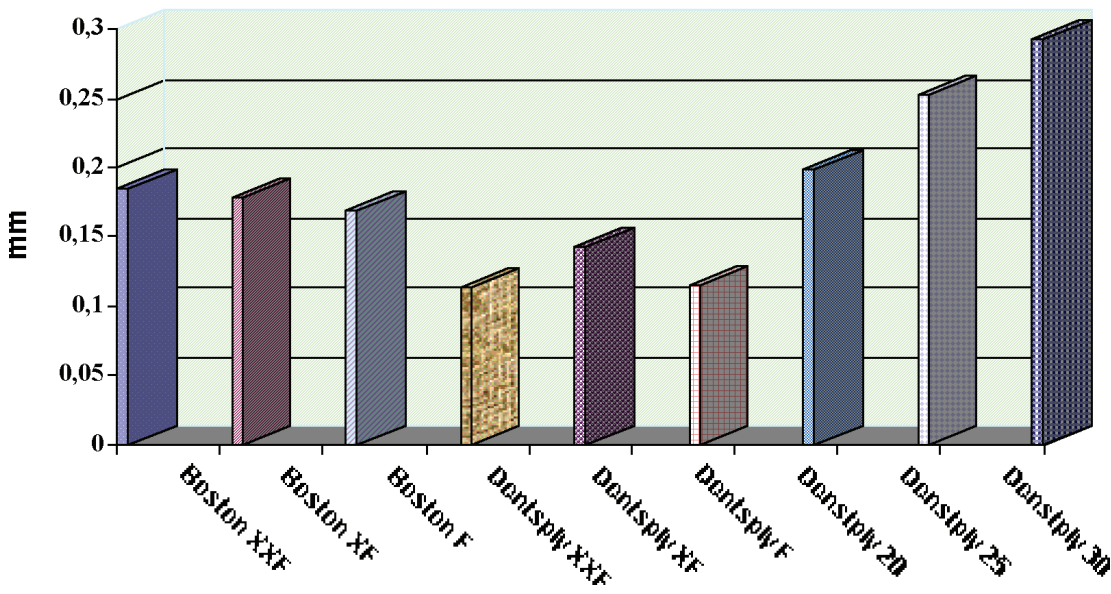

Figura 1. Media obtenida en DI según el grupo de estudio (Gutapercha).

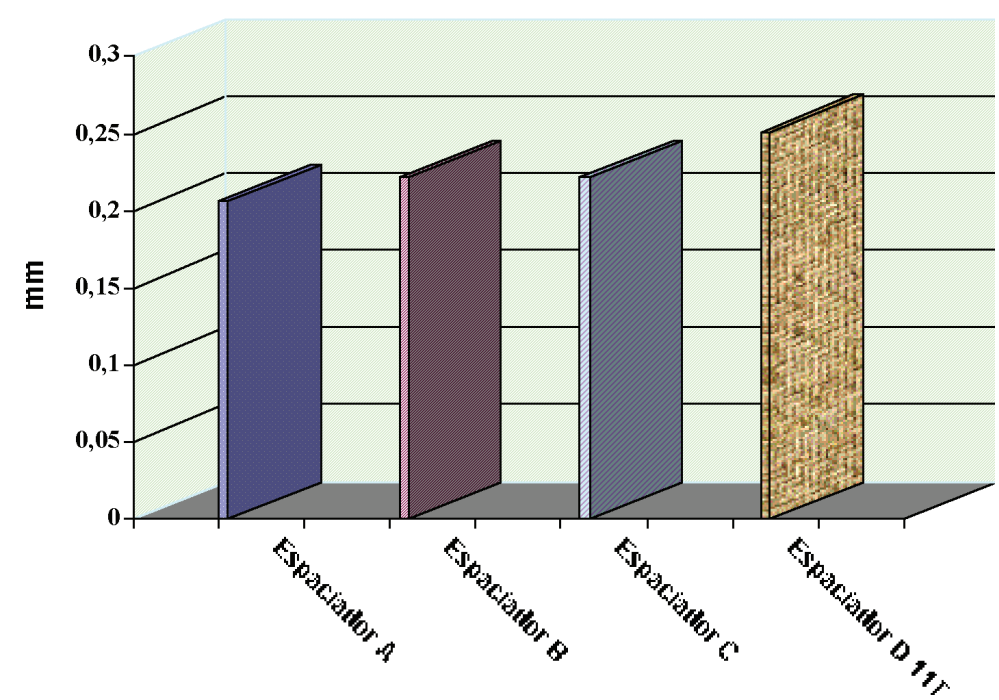

Figura 2. Media obtenida en Dl según el grupo de estudio (Espaciadores).

milímetros, a las cuales se realizó un análisis estadístico descriptivo (media aritmética, valores máximo y mínimo y desviación estándar). Sin embargo, no se aplicó ninguna prueba estadística inferencial, porque en este estudio no se comparó ningún grupo experimental entre sí, solo se observó si los diámetros de las puntas de gutapercha coincidían con los espaciadores evaluados.

\section{Resultados}

Para cada uno de los grupos se contó con diez datos o mediciones. Las tablas 2 y 3 muestran los resultados estadísticos descriptivos obtenidos en las muestras estudiadas.

En las figuras 1 y 2 se destaca la media obtenida en $D_{1}$ de los grupos constituidos por gutaperchas y espacia-

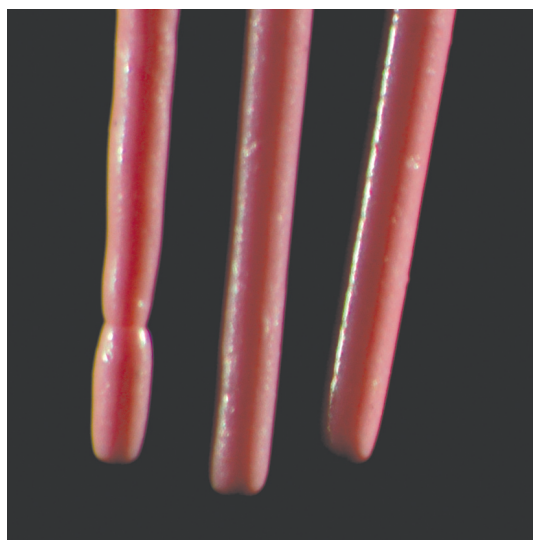

Figura 3. Tercio apical de las gutaperchas ISO 030 - 50X (DentsplyDeTrey).

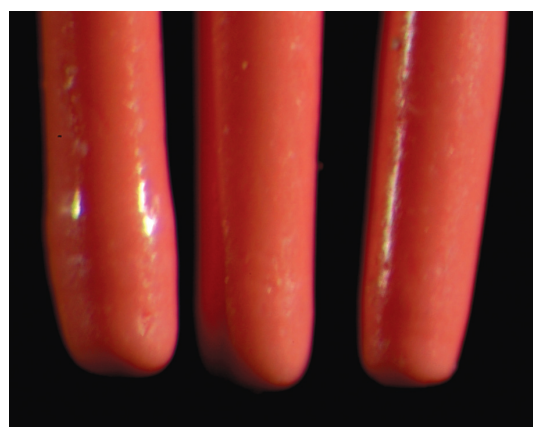

Figura 4. Tercio apical de las gutaperchas ISO $030-100 X$ (DentsplyDeTrey).

dores. Se observaron diferencias en el diámetro entre los grupos de gutapercha que pertenecen a un mismo rango (figs. 3 y 4) y entre los conos de gutapercha y los espaciadores correspondientes, de acuerdo con los criterios de la técnica de condensación lateral.

Las figuras 5 y 6 reflejan los valores de la media de los grupos de estudio correspondiente a $\mathrm{D}_{2}$, donde también se destacaron las discrepancias en el tamaño entre los grupos de gutapercha y los espaciadores.

De acuerdo con nuestros datos, los grupos Boston XXF, Boston XF, Dentsply XXF, Dentsply XF y Dentsply 20 son 


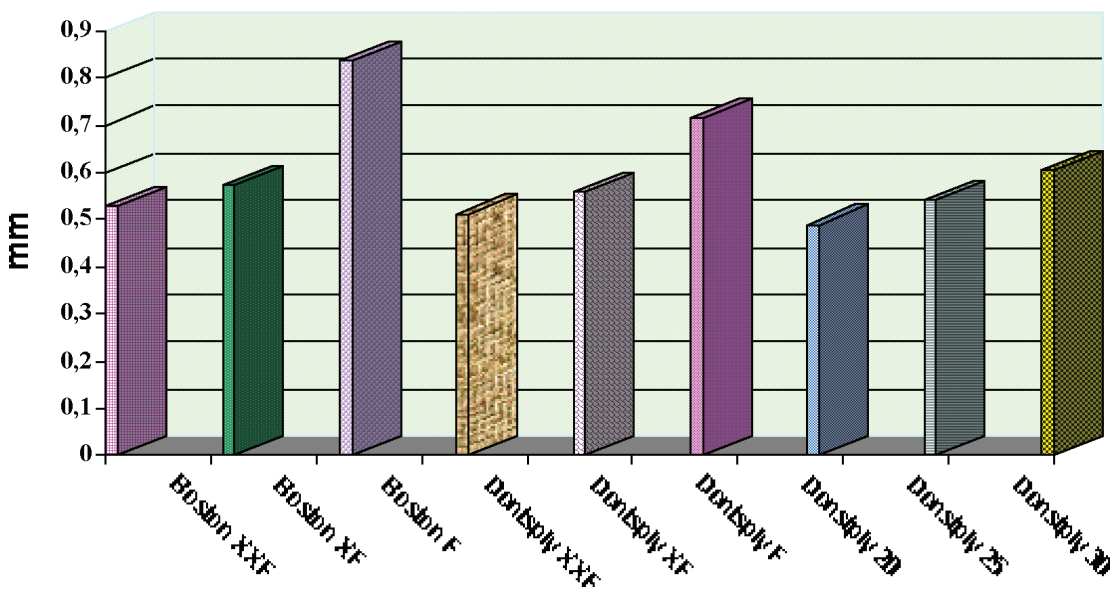

Figura 5. Media obtenida en D2 según el grupo de estudio (Gutapercha).

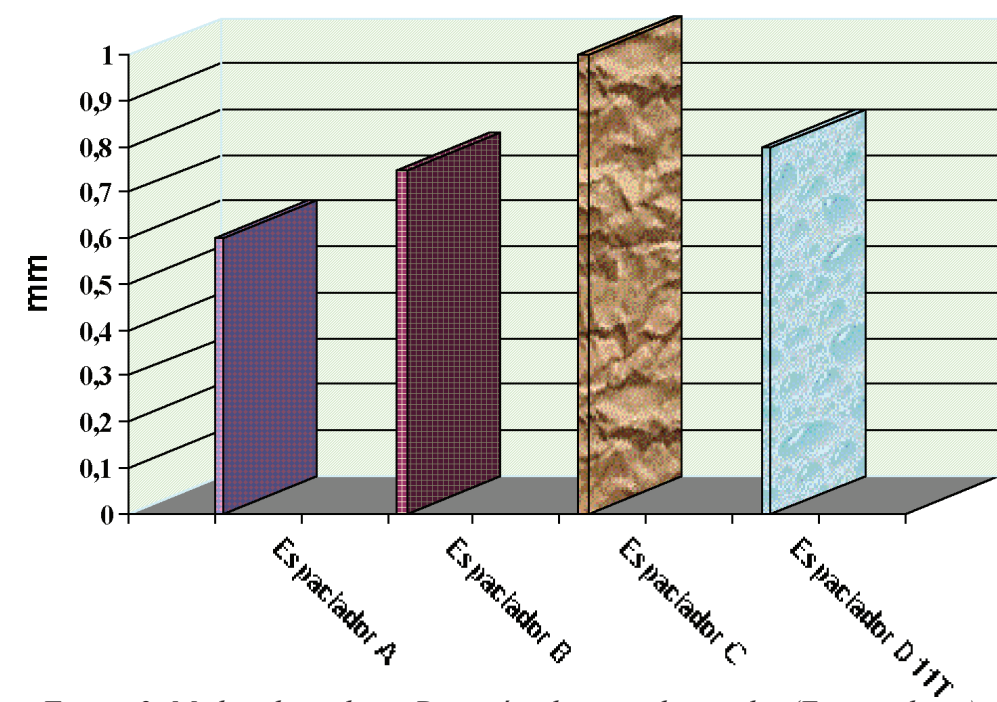

Figura 6. Media obtenida en D2 según el grupo de estudio (Espaciadores).

compatibles con los grupos Espaciador A y Espaciador B. Los grupos Boston F y Dentsply F se correlacionan de forma recíproca con el grupo Espaciador C. En los grupos Dentsply 25 y Dentsply 30, no fue posible establecer una relación con ningún grupo de espaciadores, porque su valor en $D_{1}$, se encuentra por encima del espaciador D 11T, que fue el grupo de espaciadores que obtuvo el mayor diáme- tro en D1 (fig.2 ), mientras que el grupo que ofreció el resultado más elevado en $D_{2}$ fue el grupo Espaciador $C$ (fig. 6).

Paradójicamente, los grupos Boston XF y Boston $F$ ofrecieron una media en $D_{1}$ menor que el grupo Boston XXF. Por otro lado, Ios conos accesorios de los grupos Dentsply F presentaron un tamaño medio en $D_{1}$ inferior que el grupo Dentsply $\mathrm{XF}$,

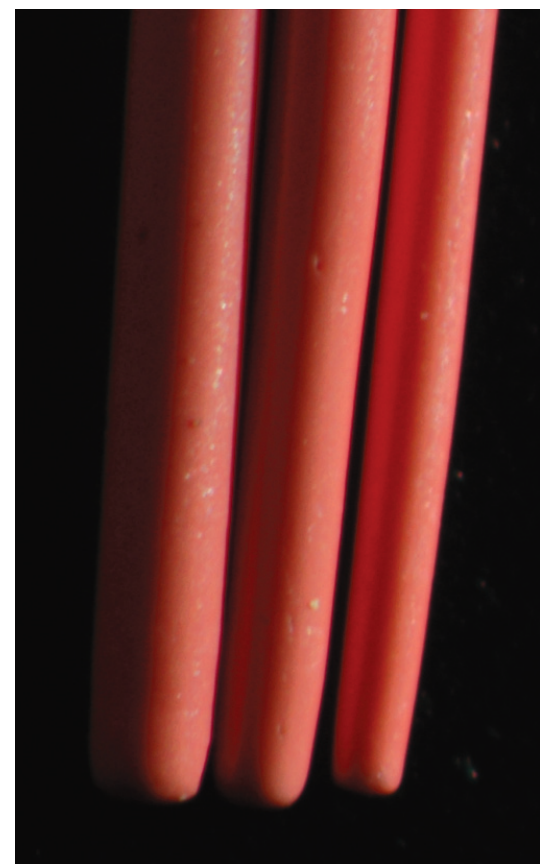

Figura 7. De derecha a izquierda: Tercio apical de las gutaperchas XXF, XF, F-50X (Dentsply-DeTrey).

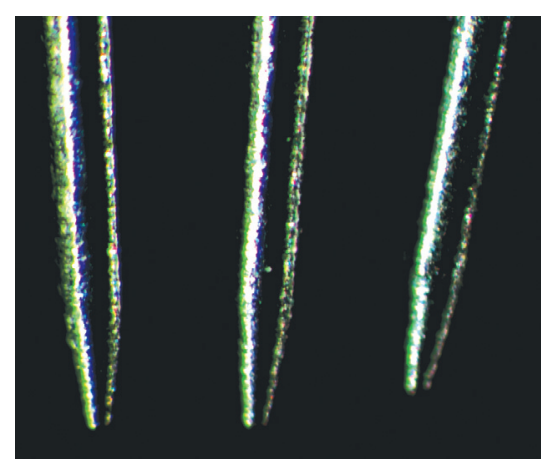

Figura 8. De derecha a izquierda: Punta de los espaciadores $A, B$ y $C-50 X$ (Dentsply-Maillefer).

incluso se observó una estrecha diferencia con el grupo Dentsply XXF (tabla 2) - (fig. 7).

Es importante destacar, que los grupos Espaciadores A y B ofrecieron las medias similares en $D_{1}$; sin embargo, presentaron diferencias importantes en $D_{2}$ (fig. 8). El grupo que pre- 
sentó la mayor desviación estándar (DE), fue Boston XF en D1 y Boston F en $D_{2}$; mientras que los grupos Espaciador A, B, C y D 11T ofrecieron una $D E$ menor, incluso en algunos casos fue igual a 0 (tablas 2 y 3 ).

\section{Discusión}

La obturación en endodoncia consiste en reemplazar el contenido del sistema de conductos radiculares por materiales inertes que los rellenen hermética y tridimensionalmente, formando así una barrera al paso de las toxinas y microorganismos para conseguir el mayor porcentaje de éxito en el tratamiento ${ }^{11}$. Según Beer y cols $^{12}$ cuanto más densa sea la obturación y más se minimicen los espacios vacíos, mejor será el pronóstico.

La técnica de condensación lateral se utiliza mundialmente, debido a su aparente simplicidad y sencillez de ejecución ${ }^{13}$. De acuerdo con Pumarola ${ }^{14}$ para obtener buenos resultados en el sellado apical y densidad de obturación se deben seleccionar conos de gutapercha que se adapten al espacio o habitáculo creado por el espaciador digital o digito - palmar. La selección de las puntas accesorias de gutapercha de la misma marca de los espaciadores tendría que mejorar la calidad de la obturación, aunque Briceño y cols $^{15^{*}}$ comprobaron la inadecuada compatibilidad entre ellos para el mismo fabricante.

Todos los materiales de obturación en endodoncia, ya sea por deficiencias en los fenómenos de adhesividad a los conductos o por las variaciones dimensionales que experimentan, dejan espacios muertos o libres, pro- piciando la filtración o percolación marginal con los sucesivos cambios biológicos que afectarán a los tejidos periapicales y al pronóstico del diente tratado ${ }^{16}$.

Por lo tanto, y según Ingle y cols ${ }^{17}$ una obturación inadecuada, incompleta o errónea del sistema de conductos radiculares es una de las causas más importantes de fracaso endodóncico. Cuando se utiliza la técnica de condensación lateral, la calidad de la obturación depende de múltiples variables, entre ellas la relación dimensional entre los conos accesorios de gutapercha y el espaciador ${ }^{18,19}$.

El espacio creado por el espaciador debería ser ocupado por un cono accesorio de calibre y conicidad proporcional. Un cono de gutapercha más grueso dejará espacios sin rellenar dentro del conducto radicular (zona apical), mientras que puntas más estrechas inevitablemente obligarán al uso de un mayor número de puntas accesorias, aumentando el tiempo de compactación ${ }^{14}$. Lo más idóneo sería utilizar conos de gutapercha que se correspondiesen con los espaciadores análogos. Es importante destacar que siempre los espaciadores deben ser de un diámetro mayor (ligeramente) comparado con el cono de gutapercha del mismo rango, lo que permite una mejor adaptación, facilita el deslizamiento de la gutapercha por el espacio creado por el espaciador, contrarresta, de alguna forma, la presencia de irregularidades y/o anfractuosidades de los conos accesorios, principalmente en las zona más cercana a $D_{1}$ (figs. 3 y 4), y permite la recuperación elástica de la gutapercha, pasados algunos segundos de la compactación.
A pesar de los esfuerzos de los fabricantes y asociaciones tales como la Asociación Dental Americana y la Organización Internacional de Estandarización, hoy en día, no es posible hablar de instrumentos estandarizados al $100 \%$, aunque se ha mejorado, en comparación con épocas pasadas $^{16}$.

La correspondencia entre conos y espaciadores puede medirse mediante la comparación de sus longitudes, conicidades y ángulos ${ }^{2 * 15^{*}, 16}$, realización de moldes de los espaciadores ${ }^{14} \mathrm{y}$ por la cantidad de gutapercha compactada en el interior del conducto ${ }^{8}$.

En nuestro estudio hemos utilizado como medio para establecer las relaciones entre los grupos de gutaperchas y espaciadores la comparación de longitudes o diámetros, al igual que las investigaciones realizadas por Zmener y cols $^{2 *}$ y Hartwell y cols . Nuestro método consistió en realizar mediciones en $D_{1}$ y $D_{2}$, sin seguir las especificaciones de la Asociación Dental Americana ( $N^{\circ}$ 57), que establece que se deben realizar las mediciones a los tres milímetros de la punta $\left(D_{3}\right)$ y a los dieciséis respectivamente ${ }^{10}$, ya que nosotros pensamos que la creación del espacio en $D_{1}$ y la adaptación del cono de gutapercha es más crítico a este nivel que en $D_{3}$.

De acuerdo con nuestros resultados, existe variabilidad dimensional entre los conos de gutapercha de un mismo tamaño, los espaciadores y las puntas de gutapercha y los espaciadores entre sí, aunque las diferencias entre este último grupo son menores (figs.1-6). Los grupos de gutapercha, en los cuales se estableció mayor correlación dimensional fueron Boston XXF, Boston XF, Dentsply XXF, Dentsply XF y Dentsply 20, mientras 
que con los grupos Dentsply 25 y Dentsply 30, no fue posible relacionarlos con ningún espaciador de los evaluados en este estudio. Este hecho se debe a que los diámetros que ofrecieron estos grupos en $D_{1}$ estaban por encima de los valores que presentaron los espaciadores.

Es importante señalar, que los grupos Espaciadores A y B ofrecieron las medias y desviaciones estándares similares en $D_{1}$; sin embargo, en $D_{2}$ sus medias presentaron diferencias importantes. Esta es la razón por la cual son los grupos de espaciadores que poseen mayor rango de compatibilidades (tablas 2 y 3 ) - (fig. 8).

Kerekes $^{7 * *}$ entre otros investigadores también ha observado discrepancias dimensionales entre diferentes marcas de gutapercha y espaciadores, no cumpliendo las especificaciones de la organización internacional de estandarización por presentar fallos de precisión en sus calibres.

Nuestro estudio se realizó en condiciones in vitro. Por lo tanto, debemos recordar que durante la obturación in vivo del sistema de conductos radiculares influyen otros factores $y$ no solo la correlación de diámetros 0 longitudes entre el espaciador y la gutapercha, tales como los movimientos rotacionales o de vaivén que genera el espaciador durante la técnica de compactación lateral, la flexibilidad del espaciador, tamaño y curvatura del conducto, posición del espa- ciador dentro del conducto, habilidad del operador, todos ellos son capaces de modificar el espacio creado por el espaciador y, por lo tanto, modificar la relación espaciador - gutapercha, a pesar de la estandarización ${ }^{4}$.

Se debe tener en cuenta que cuando varios conos ajusten en el espacio creado por el espaciador, será más adecuado seleccionar el menos cónico ya que al retirar el espaciador del conducto, en una situación clínica, los conos compactados previamente tienden a desplazarse y disminuir el espacio, impidiendo o dificultando que el cono escogido se coloque en el sitio previamente creado ${ }^{14}$.

Como se destacó con anterioridad, clínicamente hay otras variables que influyen en la calidad de la condensación de la obturación, como la distancia a la que debe llegar el espaciador. Allison y cols $^{20}$ demostraron que la filtración marginal aumenta cuando el espaciador se aleja más de un milímetro de la longitud de trabajo. Según Pumarola ${ }^{14}$ cuanto más deformable sea la gutapercha más espacio lateral podremos crear y mientras más difíciles sean los conductos (estrechos y curvos), más finos y menos cónicos deberán ser las equivalencias entre los conos y los espaciadores. Por el contrario, cuando se utilizan las técnicas corono - apicales y especialmente si se emplean sistemas rotatorios de conicidad variable, se podrán ajustar conos - espaciadores más anchos.
Es importante resaltar, que los grupos constituidos por espaciadores presentaron una desviación estándar más baja, incluso en algunos este parámetro fue igual a 0 (tabla 3); mientras el grupo que ofreció la mayor DE fue Boston F en D2. Todo ello significa que en el proceso de fabricación, es más sencillo estandarizar los instrumentos metálicos que las gutaperchas y por lo tanto es más fiable su numeración e igualdad entre instrumentos análogos.

\section{Conclusiones}

- Existen diferencias dimensionales entre los grupos de gutapercha y los espaciadores, incluso perteneciendo al mismo fabricante, las cuales podrían ser causa de desadaptaciones o vacíos durante la obturación hermética y tridimensional del sistema de conductos radiculares y propiciar un sellado apical inadecuado.

- Es más fiable la estandarización entre los instrumentos metálicos que entre las puntas de gutapercha.

- Es recomendable utilizar los conos accesorios de gutapercha de un diámetro inferior al tamaño del espaciador.

- Son necesarios más estudios en la materia para obtener un mejor conocimiento de los procesos implicados en la relación dimensional espaciador - gutapercha. 


\section{Bibliografia recomendada}

Para profundizar en la lectura de este tema, el/los autor/es considera/an interesantes los artículos que aparecen señalados del siguiente modo: *de interés * ${ }^{*}$ de especial interés.

1. Alventosa Martin J. Condensación lateral. Rev Esp Endod 1989;7(2):70-1.

2.* Zmener O, Hilu R, Scavo R. Compatibility between standardized endodontic finger spreaders and accesory gutta - percha cones. Endod Dent Traumatol 1996;12:237-9.

En este estudio se establecieron una serie de compatibilidades o correlaciones entre conos accesorios de gutapercha y espaciadores digitales.

3. Czonstkowsky M, Michanowicz A, Vazquez J. Evaluation of an injection of thermoplasticized low temperature gutta - percha using radioactive isotopes. $\mathrm{J}$ Endod 1985;11:71-4

4. Hartwell G, Barbieri S, Gerard S, Gunsolley J. Evaluation of size variation between endodontic finger spreaders and accessory gutta percha cones. J Endod 1991;17:8-11.

5. Moule A, Kellaway R, Clarkson R, et al. Variability of master gutta-percha cones. Aust Endod J 2002;28:38-43.

6 . Ingle $J$. The need for endodontic instrument standardization. Oral Surg 1955;8:1211-3.

7. ** Kerekes K. Evaluation of standardized root canal instrument and obturating points. J Endod 1979;5:145-50.

En esta investigación se compararon los instru- mentos de uso en endodoncia y conos de gutapercha con la norma ISO estándar.

8. Jerome C, Hicks M, Pelleu G. Compatibility of accessory guttapercha cones used with two types of spreaders. J Endod 1988;14:428-34.

9. Miserendino L. Report submitted to ADA/ANSI Committee on Endodontic Instrument and Materials at 42nd Annual Meeting of the American Association of Endodontics, San Diego - California, 1985.

10. American National Standards Institute/American Dental Association Specification $N^{0} \mathbf{5 7}$ for Endodontic Filling Material, 1984.

11. Chohayeb A. Evaluation of the apical condensation of gutta - percha by a tapered/calibrated spreader/plugger. J Endod 1993;19:167-9.

12. Beer R, Gängler P, Ruprecht B. Investigation of canal space occupied by gutta - percha following lateral condensation and thermomechanical condensation. Int Endod J 1987;20:271-5.

13. Valle González A. Actividades de los miembros AEDE en la práctica endodóntica. Análisis estadístico. Rev Esp Endod 1997;15:209-15.

14. Pumarola J. Compatibilidad entre espaciadores y puntas accesorias en la compactación lateral. Parte II. Rev Esp Endod 2002;20: 25865.
15.* Briseño B, Wolter D, Willershausen - Zönnchen B. Dimensional variability of nonstandardized greater taper finger spreaders with matching gutta - percha points. Int Endod 2001;34:23-8

Estos investigadores comprobaron la inadecuada compatibilidad entre los instrumentos y las puntas accesorias de gutapercha para un mismo fabricante.

16. Núñez N, Badanelli P, Martínez A. Uribe J. Variaciones físicas, diámetro y grado de conicidad en conos de gutapercha y limas tipo $\mathbf{K}$. Rev Esp Endod 1983;I(3):155-71.

17. Ingle J, Beveridge E, Glick D, Weichman J, Abou - Rass M. Modern endodontic therapy. En Ingle J, Taintor J. Endodontics. Philadelphia: Lea \& Fabiger 1985;27-37.

18. Taylor G. Advanced techniques for intracanal preparation and filling in routine endodontic therapy. Dent Clin North Am 1984;28:819-31.

19. Golberg F, Gurfinkel J, Spielberg G. Microscopic study of standardized gutta - percha points. Oral Surg Oral Med Oral Pathol 1979;47:275-6.

20. Allison D, Webber C, Walton R. The influence of the method of canal preparation on the quality of the apical and coronal obturation. J Endod 1979;5:298-304. 